\title{
Periodontal initial therapy combined with laterally positioned flap in gingival plasma cell granuloma treatment: a case report
}

\author{
Hui-Wen Chen ${ }^{1}$, Lin-Jun Shi ${ }^{2}$, Zhong-Chen Song ${ }^{1}$ \\ ${ }^{1}$ Department of Periodontology, Shanghai Ninth People's Hospital, College of Stomatology, Shanghai Jiao Tong University School of Medicine, \\ Shanghai Key Laboratory of Stomatology \& Shanghai Research Institute of Stomatology, National Clinical Research Center for Oral Diseases, \\ Shanghai, China; ${ }^{2}$ Department of Oral Mucosal Diseases, Shanghai Ninth People's Hospital, College of Stomatology, Shanghai Jiao Tong University \\ School of Medicine, Shanghai Key Laboratory of Stomatology \& Shanghai Research Institute of Stomatology, National Clinical Research Center for \\ Oral Diseases, Shanghai, China \\ Correspondence to: Zhong-Chen Song. Department of Periodontology, Shanghai Ninth People's Hospital, College of Stomatology, Shanghai Jiao Tong \\ University School of Medicine, Shanghai Key Laboratory of Stomatology \& Shanghai Research Institute of Stomatology, National Clinical Research \\ Center for Oral Diseases, 639 Zhi Zao Ju Road, Shanghai 200011, China. Email: szhongchen@sina.com.
}

\begin{abstract}
Background: Gingival plasma cell granuloma (GPCG) is a rare benign, reactive, non-neoplastic lesion characterized by the predominance of polyclonal plasma cells. Clinical diagnosis is often confused with peripheral giant cell lesion, plasmacytoma, lymphoma, and fibroma. Surgery resection predominates the treatment methods. Routine resection extends to adjacent normal tissue, causing postoperative aesthetic and soft tissue defects.

Case Description: A 58-year-old female presented an uncommon maxillary anterior gingival mass. The clinical examination revealed that it was red in color, exophytic, painless, lobulated, pedunculated, and prone to bleeding. It has no systemic symptoms. Four weeks after scaling and root planning, the lesion showed a good response to periodontal initial therapy. Then it was treated by immediate laterally positioned flap (LPF) after biopsy. The sections stained with hematoxylin and eosin showed that the subepithelial tissue contained numerous granulation tissues with lymphocytes and plasma cells infiltration, suggesting a plasma cell lesion. Immunohistochemistry staining of the tissue section showed notable cytoplasmic positivity for both $\kappa$ and $\lambda$ light chains. The pathological diagnosis was GPCG. No recurrence was seen at the 6-month follow-up.

Conclusions: The technique of immediate LPF after biopsy is speculated as an alternative to simple resection of GPCG. It could yield satisfactory esthetic outcomes and prevent postoperative root sensitivity and pain.
\end{abstract}

Keywords: Gingival plasma cell granuloma (GPCG); immunohistochemical analysis; laterally positioned flap (LPF); case report

Received: 14 July 2020; Accepted: 16 October 2020; Published: 30 March 2021.

doi: 10.21037/fomm-20-41

View this article at: http://dx.doi.org/10.21037/fomm-20-41

\section{Introduction}

Gingival plasma cell granuloma (GPCG) is a rare tumorlike lesion, which is composed of predominantly polyclonal plasma cell with few lymphocytes, histiocytes, mast cells, and eosinophils. Although plasma cell granuloma (PCG) is common in the lungs, it also occurs in other organs (1). In the head and neck, PCG is typically in the sinuses, larynx, temporal bone, tonsil, ears, and tongue $(2,3)$. The uncommon lesions in the gingiva are often mistaken as pyogenic granuloma, peripheral ossifying fibroma, or peripheral giant cell granuloma. Bhaskar et al. (4). reported the first case of GPCG in 1968. The lesions appeared as polypoid nodular tumor with smooth surface, usually accompanied with periodontal inflammation of varying degrees (5). Radiological image of the tumor showed 
infiltrated growth characteristics like malignant tumor (6). Clinical examination is vital for diagnosis, but pathology is the gold standard. Currently, most of the existing literatures are case reports without detailed treatment plan. The main treatment reported was excisional biopsy with gingivoplasty $(7,8)$. However, the disadvantages of this method are esthetic impairment of soft-tissue morphology and postoperative gingival recession.

In 1956, Grupe and Warren (9) proposed laterally positioned flap (LPF). Present evidence indicates that LPF is used as an alternative for soft-tissue root coverage, which is mainly for patients with a thick periodontal biotype (10). In 2004, Zucchelli et al. (11) showed that LPF was efficient in treating localized gingival recession defects, where complete root coverage (CRC) was accomplished in $80 \%$ of the treated sites. Chambrone et al. (12) reported that the histological results of LPF include new attachment formation and re-establishment of normal gingival structures over previously exposed root surfaces. Therefore, we speculate that an immediately LPF after biopsy may be effective in preventing postoperative root exposure.

This report shows a case of GPCG treated by periodontal initial therapy combined with an immediate LPF after biopsy. We present the following case in accordance with the CARE reporting checklist (available at https://fomm. amegroups.com/article/view/10.21037/fomm-20-41/rc).

\section{Case presentation}

A 58-year-old Chinese female was referred to the Department of Periodontology, Ninth People's Hospital Affiliated to Shanghai Jiao Tong University School of Medicine. Her chief complaint was a localized, painless overgrowth in the maxillary anterior gingiva for 6 months. The patient went to local hospital 2 months ago and took antibiotics for 1 week. However, the condition did not improve. The lesion had been progressively enlarging. She had no trauma history. She denied mouth breathing during sleep. Her medical history included hypertension without medication $(140 / 90 \mathrm{mmHg})$. She denied other systemic diseases. She had no allergies and no regular medication. Also, she presented amenorrhea for 3 years.

The clinical examination revealed an intraoral gingival mass in the labial surface of \#21 gingiva, measuring $14 \mathrm{~mm} \times 17 \mathrm{~mm} \times 10 \mathrm{~mm}$, which was red in color, exophytic, painless, lobulated, pedunculated, and prone to bleeding. Its boundary was obscure and almost beyond mucogingival junction. The gingiva of \#11 and \#12 region also had inflammatory swelling. No gingival recession were observed in \#11, \#12, and \#21. Full-mouth periodontal status was examined [plaque index: II-III; calculus index: II; gingival index: II; mean probing depth (PD): $3.4 \pm 1.5 \mathrm{~mm}$; mean clinical attachment level (CAL): $2 \pm 1.1 \mathrm{~mm}$; and percentage of bleeding on probing (BOP): $61 \%$ ]. The PD of lesion site was $6-9 \mathrm{~mm}$ and the CAL was $5-7 \mathrm{~mm}$. \#21 tooth was elongated and had I mobility (Figure 1A,B).\#11, \#12 and \#21 showed the same responses as the normal control teeth to electric pulp vitality test.

Radiographically, alveolar bone resorption in maxillary anterior teeth reached to the half of the root length (Figure 1C). Laboratory examination, including routine blood test and blood coagulation test was normal. Based on the medical history and clinical features, provisional diagnosis of PCG and periodontitis (Stage III, Grade $\mathrm{B}$, Generalized) were made. The clinical differential diagnosis included fibroma, peripheral giant cell lesion, and plasmacytoma.

Written informed consent was obtained from the patient for the publication of this case report and any accompanying images. In this case, periodontal initial therapy including full-mouth scaling and root planning by ultrasonic instruments (EMS, Master ${ }^{\circledR}$ Piezon 700, Swiss) and manual Gracey curettes (Hu-Friedy, Chicago, USA) under local anesthesia was conducted firstly. At 1 month after initial therapy, a periodontal re-evaluation was performed to confirm the suitability of periodontal surgery.

Four weeks after scaling and root planning, the lesion showed a good response to periodontal initial therapy. The boundary becomes apparent. The volume of tumor was limited to $6 \mathrm{~mm} \times 10 \mathrm{~mm} \times 3 \mathrm{~mm}$. The inflammatory gingival swelling in \#11 and \#12 also subsided (Figure 2). Gingival recession was observed after full-month subgingival scaling (\#21: $3.5 \mathrm{~mm}$; 11 : $0.5 \mathrm{~mm}$ ).

Before the surgery, a $0.12 \%$ chlorhexidine rinse was applied for intraoral antisepsis. After disinfection of the oral cavity, injection of local anesthetics (Primacaine ${ }^{\mathrm{TM}}$ adrenaline 1/100,000, Produits Dentaires Pierre Rolland, France) was performed. The gingival mass and the normal tissue within $0.5-1 \mathrm{~mm}$ around the mass were completely resected with a $\# 15 \mathrm{c}$ knife (Figure $3 A, B$ ). Then, the subjacent periosteum was removed and the root planning was performed (Figure 3C). In order to cover the denuded root, a partial-thickness flap was prepared with a distal lateral vertical releasing incision. After saline irrigation, the flap was laterally positioned at the level of the maximum root coverage (MRC), and sutured by 5-0 polyglactin 

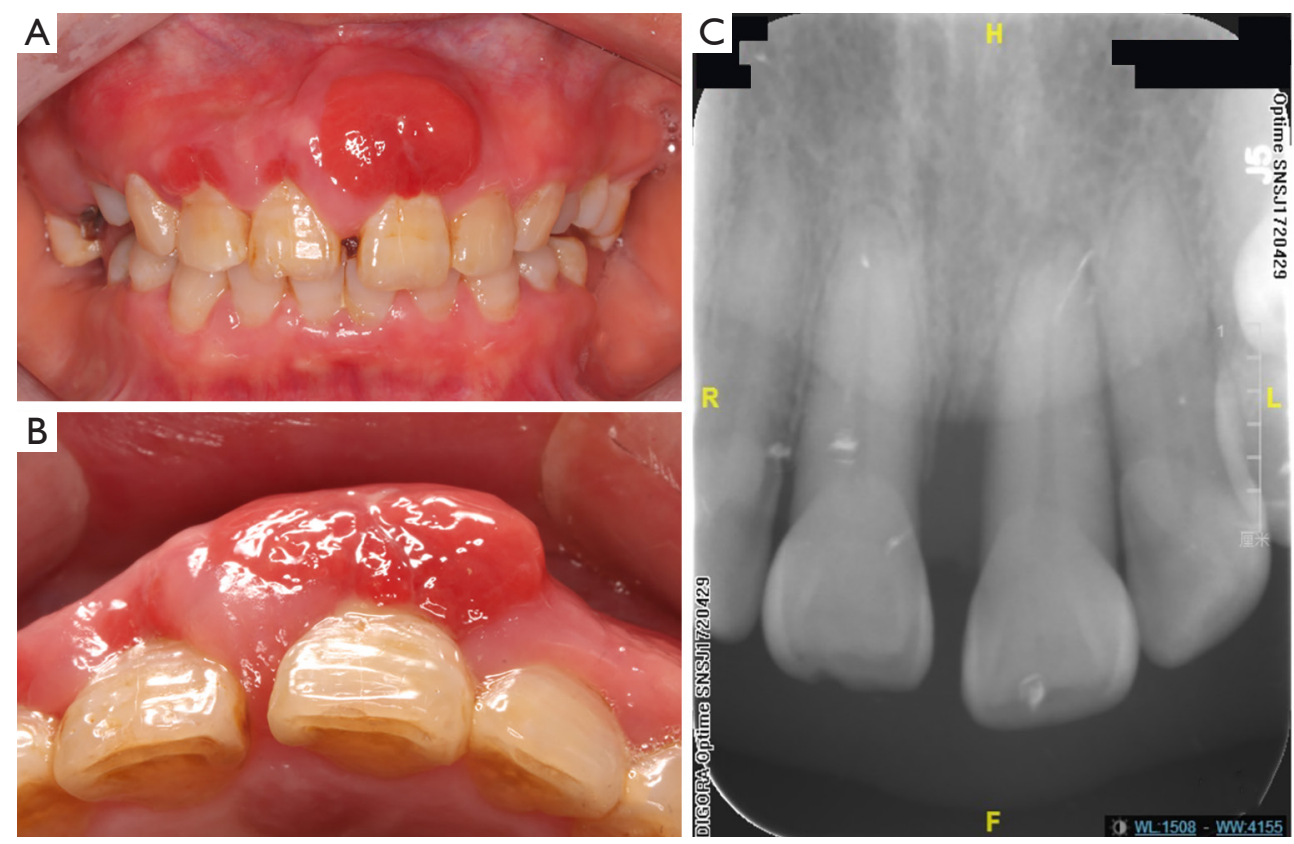

Figure 1 Pre-treatment view of the case. (A,B) Clinical examination showed an intraoral overgrowth in the labial surface of \#21 gingiva, which was red in color, exophytic, painless, lobulated, pedunculated. The boundary was obscure and almost beyond mucogingival junction. (C) Digital periapical film revealed that alveolar bone resorption in maxillary anterior teeth reached to the half of the root length.
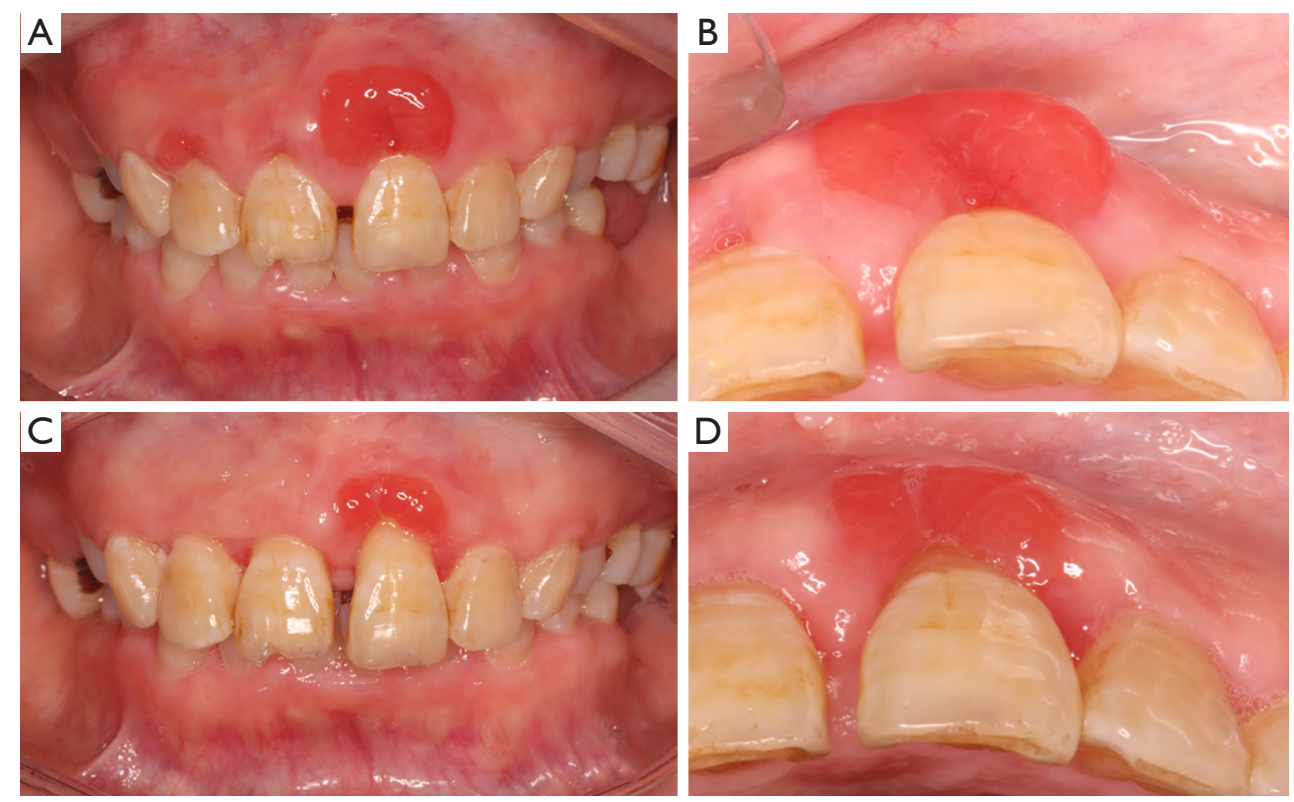

Figure 2 The outcomes of the case after periodontal initial therapy. (A,B) Appearance of mass at 2 weeks after full-month supragingival scaling; (C,D) appearance of mass at 4 weeks after second full-month subgingival scaling). 

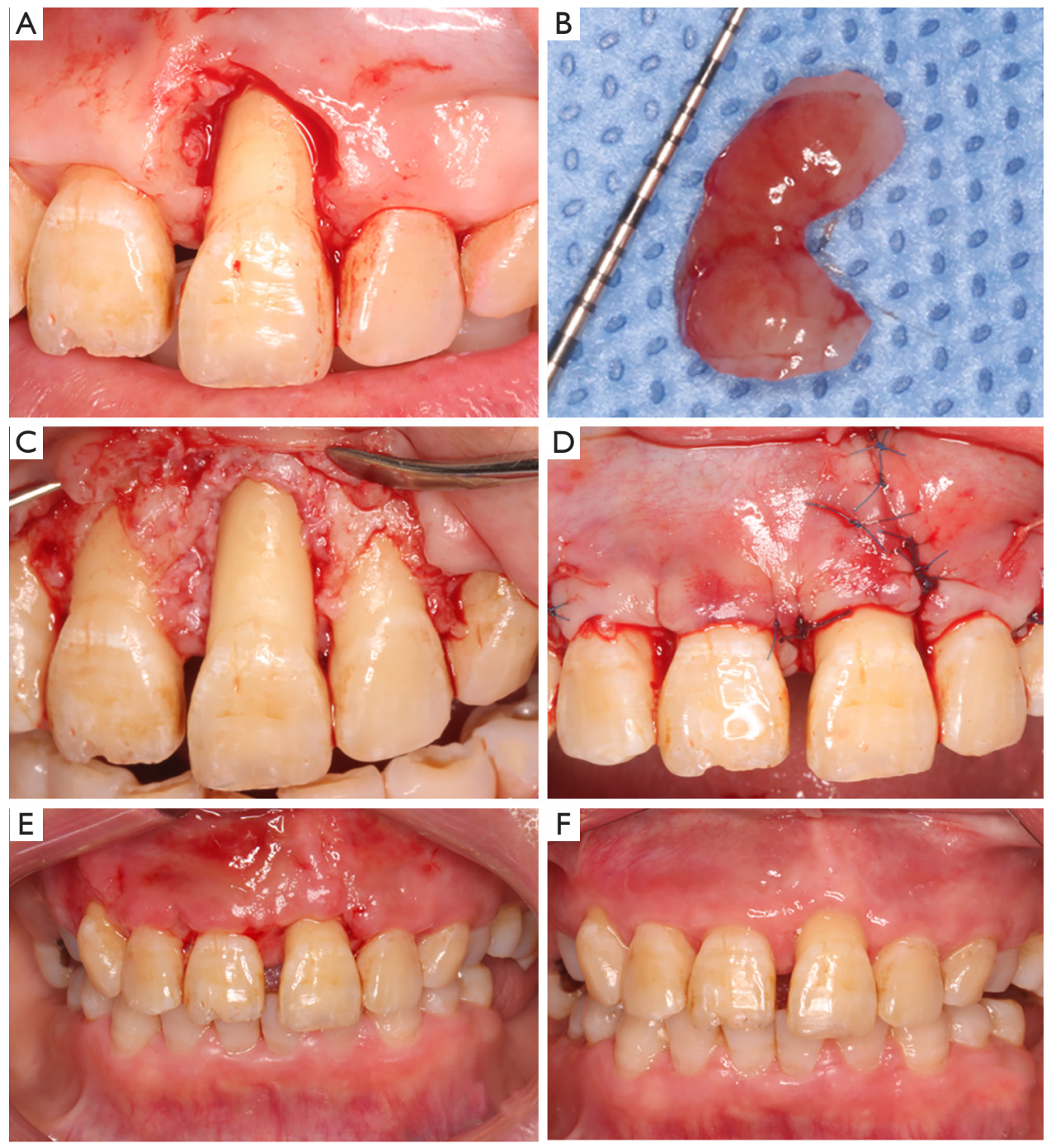

Figure 3 Intro- and Pro-operative view of the case. (A) The gingival mass and the normal tissue within $0.5-1 \mathrm{~mm}$ around the mass were completely resected; (B) the size of mass; (C) the subjacent periosteum was removed and the root planning was performed; (D) sutured LPF with 5-0 polyglactin suture materials; (E) at 2 weeks post-surgery; (F) at 6 months post-surgery. LPF, laterally positioned flap.

suture. A piece of iodoform packing was placed on the flap donor site (Figure 3D).

The patient was prescribed amoxicillin $(500 \mathrm{mg} 3$ times per day for 3 days) and ibuprofen (1 tablet per day when necessary). Also, she was advised to rinsing mouth three times daily with $0.12 \%$ chlorhexidine at least 2 weeks.

After 2 weeks post-surgery, the donor site was covered by new epithelium and stitches were removed (Figure 3E). The patient could not be recalled for periodontal examination at 3 months' interval because of the COVID-19 outbreak. No recurrence was seen at the follow-up 6 months. The gingiva was healthy. The gingival recession in \#21 was $1.5 \mathrm{~mm}$ and
\#11 was $0 \mathrm{~mm}$ (Figure $3 F$ ). The patient did not complain about any discomfort or esthetic problem. She was very satisfied with the results although the root coverage was not complete.

All procedures performed in studies involving human participants were in accordance with the ethical standards of the institutional and/or national research committee (s) and with the Helsinki Declaration (as revised in 2013).

\section{Histological and immunohistochemical result}

Histological results revealed that the mucosal epithelium 

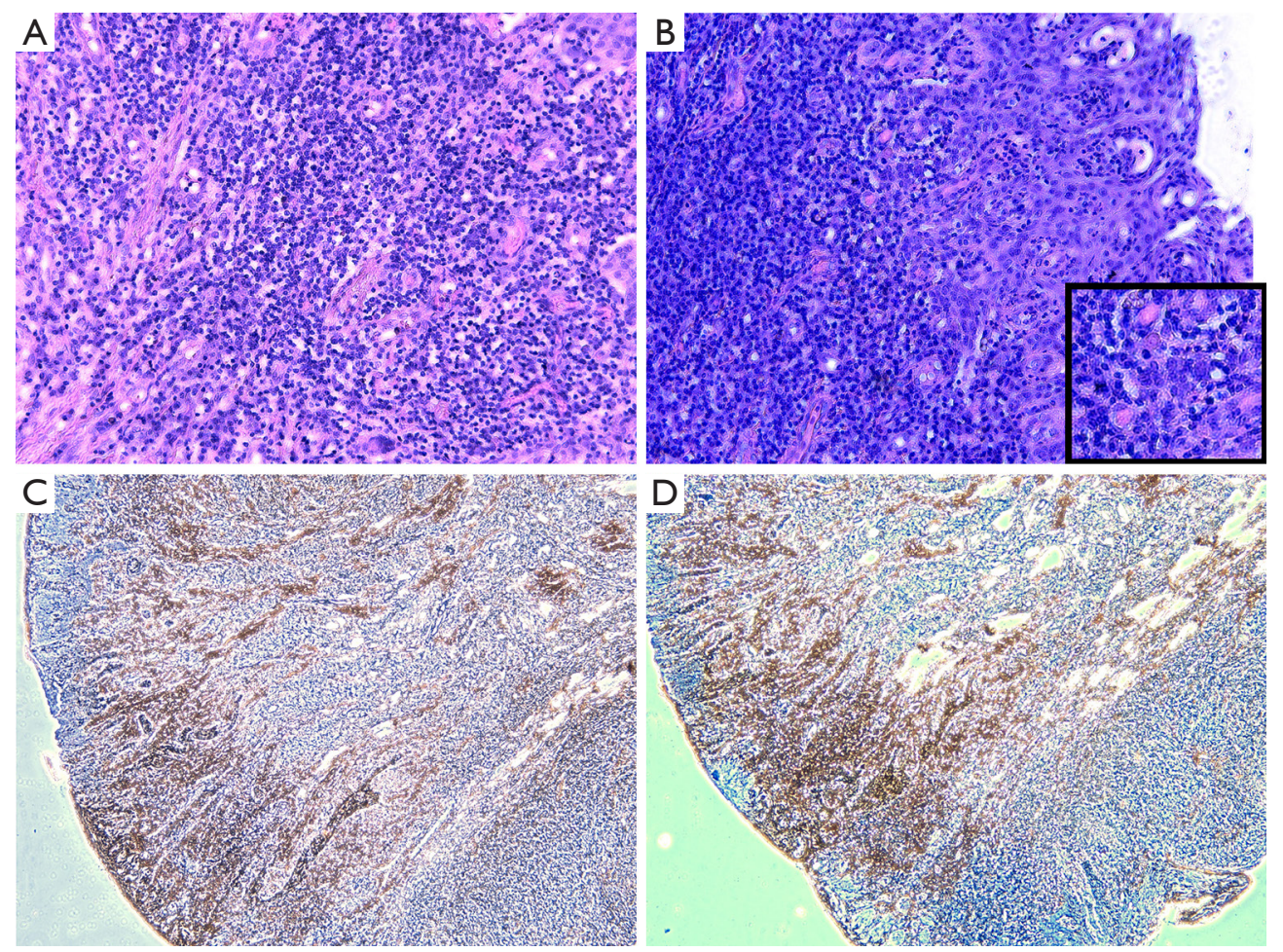

Figure 4 Histological results show diffuse infiltration of plasma cells in (A) fibrotic stroma (scale bar: $100 \mu \mathrm{m})$ and (B) Russell bodies (black box; scale bar: $100 \mu \mathrm{m})$. Immunohistochemical results show notable cytoplasmic positivity for both $\mathrm{K}$ and $\lambda$ light chains (scale bar: $50 \mu \mathrm{m})$ : (C) $\kappa$ light chain; (D) $\lambda$ light chain.

of the lesion was of the keratinized, stratified squamous type. Elongate rete pegs and areas of ulceration could be observed. Some epithelium has ulcer sites. The subepithelial tissue contained numerous granulation tissues with lymphocytes and plasma cells infiltration. The plasma cells could be identified by the high nucleus to cytoplasmic ratio, basophilic cytoplasm and their typical eccentrically placed hyperchromatic. Russell bodies (yellow circle) were observed in some areas with dense plasma cell infiltration (Figure 4A,B).

Immunohistochemical staining of $\kappa$ and $\lambda$ light chains showed a polyclonal plasma cell population (Figure $4 C, D$ ). Numerous cells were positive for CD-38, CD-3, and CD20, predominantly negative for AE1/AE3 and S100. The result ruled out tumors such as myeloma or plasmacytoma. It was confirmed that the lesion was PCG accompanied by inflammation.

\section{Discussion}

GPCG is an uncommon disease with diverse histological performance. The differential diagnosis in this case included fibroma, peripheral giant cell lesion, and plasmacytoma. Fibroma of gingiva is characterised histologically by broad interlacing fascicles of mature fibroblasts with a variable degree of collagenization (13). The peripheral giant cell granuloma is a benign lesion induced by local chronic irritation. The lesions are non-encapsulated proliferations of oval and spindle-shaped mononuclear cells (MCs) and multiple multinucleated giant cells (MGCs) in a vascular supporting stromal tissue, associated with foci of hemorrhage (14). Plasmacytoma presents diffuse sheets of neoplastic, variably differentiated, and monoclonal plasma cells in the Mitotic activity and amyloid deposition may be present and the inflammatory cells are very sparse (15).

The pathogenesis of the GPCG remains unclear. Most literatures supported that PCG is a benign inflammatory process. Some authors have suggested PCG as a hyperreactive lesion to long-standing periodontitis (5). In this case, the immunohistochemical staining of plasma cell polyclones showed that lesion is inflammatory origin. In addition, the patient was accompanied by periodontitis 
(Stage III, Grade B, Generalized). The bone resorption around \#11 and \#21 are more presumably associated with periodontitis than GPCG. Part of gingival mass might be tissue edema caused by periodontal inflammation. It could explain why this tumorous lesion responded well after periodontal initial therapy. Periodontal initial therapy is considered the most effective way to reduce periodontal inflammation. It consists of oral hygiene instructions, fullmouth scaling, and root planning if necessary. Once the initial phase of periodontal therapy is strictly completed, the periodontal inflammation is no longer present. It could help to reduce the volume of mass and make the operation field clear. This also helps to avoid residue and recurrence (16).

Surgery resection predominates the treatment methods for GPCG. A majority of literature on GPCG treatment was excisional biopsy with gingivoplasty. Simple resection of the tumor is bound to leave bare root surface that could cause root sensitivity and caries. For lesions in the anterior area like this patient, this method would result in inevitable aesthetic defects. Periodontal plastic surgery (PPS) has been used to improve the depth of gingival recession and the width of keratinized gingival tissue (17). Thus, it was applied in this case for achieving pleasing esthetics. Xie et al. (18) suggested an immediate LPF after epulis biopsy had the advantage of repairing gingival defects. Although was the gold standard for root coverage. The increase in keratinized tissue with LPF is significantly greater than CTG + CAF (19). Meanwhile, it is not necessary to create a second operation area in the palatine to reduce postoperative pain, bleeding, and other complications. This patient's gingival biotype was thick. She had a good potential donor site for harvest of a lateral sliding graft. Hwang et al. (20) reported a positive association between flap thickness and CRC. Thick biotype usually accompanies with excellent prognosis. In this case, the gingival recession type of \#21 belongs to Miller III because the maxillary incisors lacked medial contact area. Thus, the prognosis showed a result with partially root coverage. From the clinical results, it meets the preoperative expectations. There is no obvious discomfort reaction after operation and no recurrence. The effect of overall treatment is satisfactory. Under normal circumstances, invasive surgery prior to histological definition is not very appropriate. However, in our case, a provisional diagnosis of PCG combined periodontitis was made based on the typical clinical findings and good response to periodontal initial therapy. Radiologically the lesion did not show infiltrative margins like a malignant tumor. Consideration of the age and the tolerance to operation, a combination treatment of root coverage together and biopsy might provide superior clinical effectiveness. A second surgery was not necessary again. It decreased the burden on the body and economic of the patient.

\section{Conclusions}

Immunohistochemical analysis of specimens could help make accurate diagnosis of GPCG. The technique of periodontal initial therapy combined with an immediate LPF after biopsy is speculated as an alternative to simple resection of GPCG. It could yield satisfactory esthetic outcomes and prevent postoperative root sensitivity and pain.

\section{Acknowledgments}

Funding: This work was supported by the Project of Biobank from Shanghai Ninth People's Hospital, Shanghai Jiao Tong University School of Medicine (No. YBKB201905), Clinical Research Program of $9^{\text {th }}$ People's Hospital, Shanghai Jiao Tong University School of Medicine (No. JYLJ038), and Innovative research team of high-level local universities in shanghai, Oral and maxillofacial regeneration and functional restoration.

\section{Footnote}

Reporting Checklist: The authors have completed the CARE reporting checklist. Available at https://fomm.amegroups. com/article/view/10.21037/fomm-20-41/rc

Peer Review File: Available at https://fomm.amegroups.com/ article/view/10.21037/fomm-20-41/prf

Conflicts of Interest: All authors have completed the ICMJE uniform disclosure form (available at https://fomm. amegroups.com/article/view/10.21037/fomm-20-41/coif). The authors have no conflicts of interest to declare.

Ethical Statement: The authors are accountable for all aspects of the work in ensuring that questions related to the accuracy or integrity of any part of the work are appropriately investigated and resolved. All procedures performed in studies involving human participants were in accordance with the ethical standards of the institutional and/or national research committee (s) and with the Helsinki Declaration (as revised in 2013). Written informed 
consent was obtained from the patient for publication of this study and any accompanying images.

Open Access Statement: This is an Open Access article distributed in accordance with the Creative Commons Attribution-NonCommercial-NoDerivs 4.0 International License (CC BY-NC-ND 4.0), which permits the noncommercial replication and distribution of the article with the strict proviso that no changes or edits are made and the original work is properly cited (including links to both the formal publication through the relevant DOI and the license). See: https://creativecommons.org/licenses/by-nc-nd/4.0/.

\section{References}

1. Garg RA, Kumar R, Krashak R. Plasma cell granuloma of the lung. Indian Pediatr 1992;29:1556-9.

2. Vahedi A, Moya-Plana A, Guyot S, et al. Plasma Cell Granuloma of the Jaw and the Infratemporal Fossa: A Clinical Case. J Oral Maxillofac Surg 2018;76:363-7.

3. Soares J, Nunes JF, Sacadura J. Plasma cell granuloma of the tongue. Report of a case. Histol Histopathol 1987;2:199-201.

4. Bhaskar SN, Levin MP, Frisch J. Plasma cell granuloma of periodontal tissues. Report of 45 cases. Periodontics 1968;6:272-6.

5. Jhingta PK, Mardi K, Sharma D, et al. An Enigmatic Clinical Presentation of Plasma Cell Granuloma of the Oral Cavity. Contemp Clin Dent 2018;9:132-6.

6. Fang JC, Dym H. Myofibroblastic tumor of the oral cavity. A rare clinical entity. N Y State Dent J 2004;70:28-30.

7. Akdoğan N, Yalçın B, Gür Aksoy G, et al. A Case of Plasma Cell Granuloma Located on the Gingiva. Am J Dermatopathol 2017;39:393-6.

8. Jeyaraj P, Bandyopadhyay TK, Naresh N, et al. Value of Immunohistochemistry in Diagnosing a Rare Case of Maxillofacial Plasma Cell Granuloma Masquerading as a Gingival Epulis. J Maxillofac Oral Surg 2015;14:40-5.

9. Grupe HE, Warren RF. Repair of Gingival Defects by a

doi: $10.21037 /$ fomm-20-41

Cite this article as: Chen HW, Shi LJ, Song ZC. Periodontal initial therapy combined with laterally positioned flap in gingival plasma cell granuloma treatment: a case report. Front Oral Maxillofac Med 2021;3:9.
Sliding Flap Operation. J Periodontol 1956;27:92-5.

10. Chambrone L, de Castro Pinto RCN, Chambrone LA. The concepts of evidence-based periodontal plastic surgery: Application of the principles of evidence-based dentistry for the treatment of recession-type defects. Periodontol 2000 2019;79:81-106.

11. Zucchelli G, Cesari C, Amore C, et al. Laterally Moved, Coronally Advanced Flap: A Modified Surgical Approach for Isolated Recession-Type Defects. J Periodontol 2004;75:1734-41.

12. Chambrone L, Chambrone LA, Tatakis DN, et al. Wound Healing of the Laterally Positioned Flap: A Histomorphometric Assessment. Int J Periodontics Restorative Dent 2015;35:785-92.

13. Pandav AB, Gosavi AV, Lanjewar DN, et al. Gingival plasma cell granuloma. Dent Res J (Isfahan) 2012;9:816-20.

14. Boşca AB, Şovrea AS, Miclăuş V, et al. Diagnostic and therapeutic approaches in oral cavity granulomas based on new data concerning their origin and pathogenesis. Rom J Morphol Embryol 2018;59:679-90.

15. Mumtaz S. Plasmacytoma. Oral Oncol 2018;82:196-7.

16. Jiao J, Shi D, Cao ZQ, et al. Effectiveness of non-surgical periodontal therapy in a large Chinese population with chronic periodontitis. J Clin Periodontol 2017;44:42-50.

17. Scheyer ET, Sanz M, Dibart S, et al. Periodontal Soft Tissue Non-Root Coverage Procedures: A Consensus Report From the AAP Regeneration Workshop. J Periodontol 2015;86:S73-6.

18. Xie YF, Shu R, Qian JL, et al. Esthetic Management of Mucogingival Defects After Excision of Epulis Using Laterally Positioned Flaps. Cell Biochem Biophys; 71:1005-10.

19. Ricci G, Silvestri M, Rasperini G, et al. Root coverage: a clinical/statistical comparison between subpedicle connective tissue graft and laterally positioned full thickness flaps. J Esthet Dent 1996;8:66-73.

20. Hwang D, Wang HL. Flap thickness as a predictor of root coverage: a systematic review. J Periodontol 2006;77:1625-34. 